\title{
Metrical characterization of super-reflexivity and linear type of Banach spaces
}

\author{
FLORENT BAUDIER
}

\begin{abstract}
We prove that a Banach space $X$ is not super-reflexive if and only if the hyperbolic infinite tree embeds metrically into $X$. We improve one implication of J.Bourgain's result who gave a metrical characterization of superreflexivity in Banach spaces in terms of uniform embeddings of the finite trees. A characterization of the linear type for Banach spaces is given using the embedding of the infinite tree equipped with the metrics $d_{p}$ induced by the $\ell_{p}$ norms.
\end{abstract}

Mathematics Subject Classification (2000). 46B20, 51F99.

Keywords. Super-reflexivity, trees, linear type, metric embedding.

1. Introduction and Notation. We fix some notation and recall basic results.

Let $(M, d)$ and $(N, \delta)$ be two metric spaces and an injective map $f: M \rightarrow N$. Following [11], we define the distortion of $f$ to be

$$
\operatorname{dist}(f):=\|f\|_{L i p}\left\|f^{-1}\right\|_{\text {Lip }}=\sup _{x \neq y \in M} \frac{\delta(f(x), f(y))}{d(x, y)} \cdot \sup _{x \neq y \in M} \frac{d(x, y)}{\delta(f(x), f(y))} .
$$

If $\operatorname{dist}(f)$ is finite, we say that $f$ is a metric embedding, or simply an embedding of $M$ into $N$.

And if there exists an embedding $f$ from $M$ into $N$, with $\operatorname{dist}(f) \leq C$, we use the notation $M \stackrel{C}{\hookrightarrow} N$.

Denote $\Omega_{0}=\{\varnothing\}$, the root of the tree. Let $\Omega_{n}=\{-1,1\}^{n}, T_{n}=\bigcup_{i=0}^{n} \Omega_{i}$ and $T=\bigcup_{n=0}^{\infty} \Omega_{n}$. Thus $T_{n}$ is the finite tree with $n$ levels and $T$ the infinite tree.

For $\varepsilon, \varepsilon^{\prime} \in T$, we note $\varepsilon \leq \varepsilon^{\prime}$ if $\varepsilon^{\prime}$ is an extension of $\varepsilon$.

Denote $|\varepsilon|$ the length of $\varepsilon$; i.e the numbers of nodes of $\varepsilon$. We define the hyperbolic distance between $\varepsilon$ and $\varepsilon^{\prime}$ by $\rho\left(\varepsilon, \varepsilon^{\prime}\right)=|\varepsilon|+\left|\varepsilon^{\prime}\right|-2|\delta|$, where $\delta$ is the greatest common ancestor of $\varepsilon$ and $\varepsilon^{\prime}$. The metric on $T_{n}$, is the restriction of $\rho$. 
For a Banach space $X$, we denote $B_{X}$ its closed unit ball, and $X^{*}$ its dual space.

$T$ embeds isometrically into $\ell_{1}(\mathbb{N})$ in a trivial way. Actually, let $\left(e_{\varepsilon}\right)_{\varepsilon \in T}$ be the canonical basis of $\ell_{1}(T)$ ( $T$ is countable), then the embedding is given by $\varepsilon \mapsto \sum_{s \leq \varepsilon} e_{s}$

Aharoni proved in [1] that every separable metric space embeds into $c_{0}$, so $T$ does.

The main result of this article is an improvement of Bourgain's metrical characterization of super-reflexivity. Bourgain proved in [2] that $X$ is not super-reflexive if and only if the finite trees $T_{n}$ uniformly embed into $X$ (i.e with embedding constants independent of $n$ ). Obviously if $T$ embeds into $X$ then the $T_{n}^{\prime} s$ embed uniformly into $X$ and $X$ is not super-reflexive, but if $X$ is not super-reflexive we did not know whether the infinite tree $T$ embeds into $X$. In this paper, we prove that it is indeed the case :

Theorem 1.1. Let $X$ be a non super-reflexive Banach space, then $(T, \rho)$ embeds into $X$.

The proof of the direct part of Bourgain's Theorem essentially uses James' characterization of super-reflexivity (see [7]) and an enumeration of the finite trees $T_{n}$. We recall James' Theorem:

Theorem 1.2 (James). Let $0<\theta<1$ and $X$ a non super-reflexive Banach space, then: $\forall n \in \mathbb{N}, \exists x_{1}, x_{2}, \ldots, x_{n} \in B_{X}, \exists x_{1}^{*}, x_{2}^{*}, \ldots, x_{n}^{*} \in B_{X^{*}}$ s.t:

$$
\begin{array}{ll}
x_{k}^{*}\left(x_{j}\right)=\theta & \forall k<j \\
x_{k}^{*}\left(x_{j}\right)=0 & \forall k \geq j
\end{array}
$$

2. Metrical characterization of super-reflexivity. The main obstruction to the embedding of $T$ into any non-super-reflexive Banach space $X$ is the finiteness of the sequences in James' characterization. How, with a sequence of Bourgain's type embedding, can we construct a single embedding from $T$ into $X$ ?

In [13], Ribe shows in particular, that $\bigoplus_{2} l_{p_{n}}$ and $\left(\bigoplus_{2} l_{p_{n}}\right) \bigoplus l_{1}$ are uniformly homeomorphic, where $\left(p_{n}\right)_{n}$ is a sequence of numbers such that $p_{n}>1$, and $p_{n}$ tends to 1 . But $T$ embeds into $l_{1}$, hence via the uniform homeomorphism $T$ embeds into $\bigoplus_{2} l_{p_{n}}$. However $T$ does not embed into any $l_{p_{n}}$ (they are super-reflexive).

The problem solved in the next theorem, inspired in part by Ribe's proof, is to construct a subspace with a Schauder decomposition $\bigoplus F_{n}$ where $T_{2^{n+1}}$ embeds into $F_{n}$ and to repast properly the embeddings in order to obtain the desired embedding.

Proof of Theorem 1.1. Let $\left(\varepsilon_{i}\right)_{i \geq 0}$, a sequence of positive real numbers such that $\prod_{i \geq 0}\left(1+\varepsilon_{i}\right) \leq 2$, and fix $0<\theta<1$. Let $k_{n}=2^{2^{n+1}+1}-1$.

First we construct inductively a sequence $\left(F_{n}\right)_{n \geq 0}$ of subspaces of $X$, which is a Schauder finite dimensional decomposition of a subspace of $X$ s.t the projection 
from $\bigoplus_{i=0}^{q} F_{i}$ onto $\bigoplus_{i=0}^{p} F_{i}$, with kernel $\bigoplus_{i=p+1}^{q} F_{i}$ (with $p<q$ ) is of norm at most $\prod_{i=p}^{q-1}\left(1+\varepsilon_{i}\right)$, and sequences

s.t:

$$
\begin{aligned}
& x_{n, 1}, x_{n, 2}, \ldots, x_{n, k_{n}} \in B_{F_{n}} \\
& x_{n, 1}^{*}, x_{n, 2}^{*}, \ldots, x_{n, k_{n}}^{*} \in B_{X^{*}}
\end{aligned}
$$

$$
\begin{array}{ll}
x_{n, k}^{*}\left(x_{n, j}\right)=\theta & \forall k<j \\
x_{n, k}^{*}\left(x_{n, j}\right)=0 & \forall k \geq j .
\end{array}
$$

Denote $\Phi_{n}: T_{n} \rightarrow\left\{1,2, \ldots, 2^{n+1}-1\right\}$ the enumeration of $T_{n}$ following the lexicographic order. It is an enumeration of $T_{n}$ such that any pair of segments in $T_{n}$ starting at incomparable nodes (with respect to the tree ordering $\leq$ ) are mapped inside disjoint intervals.

Let $\Psi_{n}=\Phi_{2^{n+1}}$ and $\Gamma_{n}=T_{2^{n+1}}$.

$X$ is non super-reflexive, hence from James' Theorem:

$\exists x_{0,1}, x_{0,2}, \ldots, x_{0,7} \in B_{X}, \exists x_{0,1}^{*}, x_{0,2}^{*}, \ldots, x_{0,7}^{*} \in B_{X^{*}}$ s.t:

$$
\begin{array}{ll}
x_{0, k}^{*}\left(x_{0, j}\right)=\theta & \forall k<j \\
x_{0, k}^{*}\left(x_{0, j}\right)=0 & \forall k \geq j .
\end{array}
$$

$\Gamma_{0}=T_{2}$ embeds into $X$ via the embedding $f_{0}(\varepsilon)=\sum_{s \leq \varepsilon} x_{0, \Psi_{0}(s)}$ (see [2]).

Let $F_{0}=\operatorname{Span}\left\{x_{0,1}, \ldots, x_{0,7}\right\}$, then $\operatorname{dim}\left(F_{0}\right)<\infty$.

Suppose that $F_{p}$ and

$$
\begin{aligned}
& x_{p, 1}, x_{p, 2}, \ldots, x_{p, k_{p}} \in B_{F_{p}} \\
& x_{p, 1}^{*}, x_{p, 2}^{*}, \ldots, x_{p, k_{p}}^{*} \in B_{X^{*}}
\end{aligned}
$$

verifying the required conditions, are constructed for all $p \leq n$.

We apply Mazur's Lemma (see [9] page 4) to the finite dimensional subspace $\bigoplus_{i=0}^{n} F_{i}$ of $X$. Thus there exists $Y_{n} \subset X$ such that $\operatorname{dim}\left(X / Y_{n}\right)<\infty$ and:

$$
\|x\| \leq\left(1+\varepsilon_{n}\right)\|x+y\|, \forall(x, y) \in \bigoplus_{i=0}^{n} F_{i} \times Y_{n} .
$$

But $Y_{n}$ is of finite codimension in $X$, hence is not super-reflexive. From James' Theorem and Hahn-Banach Theorem:

s.t:

$$
\begin{aligned}
& \exists x_{n+1,1}, x_{n+1,2}, \ldots, x_{n+1, k_{n+1}} \in B_{Y_{n}}, \\
& \exists x_{n+1,1}^{*}, x_{n+1,2}^{*}, \ldots, x_{n+1, k_{n+1}}^{*} \in B_{X^{*}},
\end{aligned}
$$

$$
\begin{array}{ll}
x_{n+1, k}^{*}\left(x_{n+1, j}\right)=\theta & \forall k<j \\
x_{n+1, k}^{*}\left(x_{n+1, j}\right)=0 & \forall k \geq j .
\end{array}
$$

$\Gamma_{n+1}$ embeds into $Y_{n}$ via the embedding $f_{n+1}(\varepsilon)=\sum_{s \leq \varepsilon} x_{n+1, \Psi_{n+1}(s)}$.

Let $F_{n+1}=\operatorname{Span}\left\{x_{n+1, j} ; 1 \leq j \leq k_{n+1}\right\}$, then $\operatorname{dim}\left(F_{n+1}\right)<\infty$, which achieves the induction. 
Now define the following projections:

Let, $P_{n}$ the projection from $\overline{\operatorname{Span}}\left(\bigcup_{i=0}^{\infty} F_{i}\right)$ onto $F_{0} \bigoplus \cdots \bigoplus F_{n}$ with kernel $\overline{\operatorname{Span}}\left(\bigcup_{i=n+1}^{\infty} F_{i}\right)$.

It is easy to show that $\left\|P_{n}\right\| \leq \prod_{i=n}^{\infty}\left(1+\varepsilon_{i}\right) \leq 2$.

We denote now $\Pi_{0}=P_{0}$ and $\Pi_{n}=P_{n}-P_{n-1}$ for $n \geq 1$. We have that $\left\|\Pi_{n}\right\| \leq 4$.

From Bourgain's construction, for all $n$ :

$$
\frac{\theta}{3} \rho\left(\varepsilon, \varepsilon^{\prime}\right) \leq\left\|f_{n}(\varepsilon)-f_{n}\left(\varepsilon^{\prime}\right)\right\| \leq \rho\left(\varepsilon, \varepsilon^{\prime}\right)
$$

where $f_{n}$ denotes the Bourgain's type embedding from $\Gamma_{n}$ in $F_{n}$, i.e $f_{n}(\varepsilon)=$ $\sum_{s \leq \varepsilon} x_{n, \Psi_{n}(s)}$.

Note that:

$$
\forall n, \forall \varepsilon \in \Gamma_{n}\left\|f_{n}(\varepsilon)\right\| \leq|\varepsilon|
$$

Now we define our embedding.

Let

$$
\begin{aligned}
f: T & \rightarrow Y=\overline{\operatorname{Span}}\left(\bigcup_{i=0}^{\infty} F_{i}\right) \subset X \\
\varepsilon & \mapsto \lambda f_{n}(\varepsilon)+(1-\lambda) f_{n+1}(\varepsilon), \text { if } 2^{n} \leq|\varepsilon| \leq 2^{n+1}
\end{aligned}
$$

where,

$$
\lambda=\frac{2^{n+1}-|\varepsilon|}{2^{n}}
$$

We will prove that:

$$
\forall \varepsilon, \varepsilon^{\prime} \in T \frac{\theta}{24} \rho\left(\varepsilon, \varepsilon^{\prime}\right) \leq\left\|f(\varepsilon)-f\left(\varepsilon^{\prime}\right)\right\| \leq 9 \rho\left(\varepsilon, \varepsilon^{\prime}\right) .
$$

Remark 2.1. We have $\frac{\theta}{24}|\varepsilon| \leq\|f(\varepsilon)\| \leq|\varepsilon|$.

First of all, we show that $f$ is 9-Lipschitz.

We can suppose that $0<|\varepsilon| \leq\left|\varepsilon^{\prime}\right|$ w.r.t remark 2.1.

If $|\varepsilon| \leq \frac{1}{2}\left|\varepsilon^{\prime}\right|$ then:

$$
\rho\left(\varepsilon, \varepsilon^{\prime}\right) \geq\left|\varepsilon^{\prime}\right|-|\varepsilon| \geq \frac{|\varepsilon|+\left|\varepsilon^{\prime}\right|}{3}
$$

Hence,

$$
\left\|f(\varepsilon)-f\left(\varepsilon^{\prime}\right)\right\| \leq 3 \rho\left(\varepsilon, \varepsilon^{\prime}\right) .
$$

If $\frac{1}{2}\left|\varepsilon^{\prime}\right|<|\varepsilon| \leq\left|\varepsilon^{\prime}\right|$, we have two different cases to consider. 
1) if $2^{n} \leq|\varepsilon| \leq\left|\varepsilon^{\prime}\right|<2^{n+1}$.

Then, let

$$
\begin{aligned}
& \lambda=\frac{2^{n+1}-|\varepsilon|}{2^{n}} \text { and } \lambda^{\prime}=\frac{2^{n+1}-\left|\varepsilon^{\prime}\right|}{2^{n}} . \\
\left\|f(\varepsilon)-f\left(\varepsilon^{\prime}\right)\right\|= & \left\|\lambda f_{n}(\varepsilon)-\lambda^{\prime} f_{n}\left(\varepsilon^{\prime}\right)+(1-\lambda) f_{n+1}(\varepsilon)-\left(1-\lambda^{\prime}\right) f_{n+1}\left(\varepsilon^{\prime}\right)\right\| \\
\leq & \lambda\left\|f_{n}(\varepsilon)-f_{n}\left(\varepsilon^{\prime}\right)\right\|+\left|\lambda-\lambda^{\prime}\right|\left(\left\|f_{n}\left(\varepsilon^{\prime}\right)\right\|+\left\|f_{n+1}\left(\varepsilon^{\prime}\right)\right\|\right) \\
& +(1-\lambda)\left\|f_{n+1}(\varepsilon)-f_{n+1}\left(\varepsilon^{\prime}\right)\right\| \\
\leq & \rho\left(\varepsilon, \varepsilon^{\prime}\right)+2 \rho\left(\varepsilon, \varepsilon^{\prime}\right)+2 \rho\left(\varepsilon, \varepsilon^{\prime}\right) \\
\leq & 5 \rho\left(\varepsilon, \varepsilon^{\prime}\right), \\
\text { because }\left\|f_{n}\left(\varepsilon^{\prime}\right)\right\|<2^{n+1},\left\|f_{n+1}\left(\varepsilon^{\prime}\right)\right\|<2^{n+1} \text { and, } & \left|\lambda-\lambda^{\prime}\right|=\frac{\left|\varepsilon^{\prime}\right|-|\varepsilon|}{2^{n}} \leq \frac{\rho\left(\varepsilon, \varepsilon^{\prime}\right)}{2^{n}} .
\end{aligned}
$$

2) if $2^{n} \leq|\varepsilon| \leq 2^{n+1} \leq\left|\varepsilon^{\prime}\right|<2^{n+2}$.

Then, let

$$
\begin{aligned}
& \lambda=\frac{2^{n+1}-|\varepsilon|}{2^{n}} \text { and } \lambda^{\prime}=\frac{2^{n+2}-\left|\varepsilon^{\prime}\right|}{2^{n+1}} . \\
\left\|f(\varepsilon)-f\left(\varepsilon^{\prime}\right)\right\|= & \left\|\lambda f_{n}(\varepsilon)+(1-\lambda) f_{n+1}(\varepsilon)-\lambda^{\prime} f_{n+1}\left(\varepsilon^{\prime}\right)-\left(1-\lambda^{\prime}\right) f_{n+2}\left(\varepsilon^{\prime}\right)\right\| \\
\leq & \lambda\left(\left\|f_{n}(\varepsilon)\right\|+\left\|f_{n+1}(\varepsilon)\right\|\right)+\left(1-\lambda^{\prime}\right)\left(\left\|f_{n+1}\left(\varepsilon^{\prime}\right)\right\|+\left\|f_{n+2}\left(\varepsilon^{\prime}\right)\right\|\right) \\
& +\left\|f_{n+1}(\varepsilon)-f_{n+1}\left(\varepsilon^{\prime}\right)\right\| \\
\leq & \rho\left(\varepsilon, \varepsilon^{\prime}\right)+2 \lambda|\varepsilon|+2\left(1-\lambda^{\prime}\right)\left|\varepsilon^{\prime}\right| \\
\leq & 9 \rho\left(\varepsilon, \varepsilon^{\prime}\right),
\end{aligned}
$$

because,

$$
\lambda \leq \frac{\rho\left(\varepsilon, \varepsilon^{\prime}\right)}{2^{n}}, \quad \text { so } \lambda|\varepsilon| \leq 2 \rho\left(\varepsilon, \varepsilon^{\prime}\right) .
$$

Similarly

$$
1-\lambda^{\prime}=\frac{\left|\varepsilon^{\prime}\right|-2^{n+1}}{2^{n+1}} \leq \frac{\rho\left(\varepsilon, \varepsilon^{\prime}\right)}{2^{n+1}} \text { and }\left(1-\lambda^{\prime}\right)\left|\varepsilon^{\prime}\right| \leq 2 \rho\left(\varepsilon, \varepsilon^{\prime}\right) .
$$

Finally, $f$ is 9-Lipschitz.

Now we deal with the minoration.

In our next discussion, whenever $|\varepsilon|$ (respectively $\left.\left|\varepsilon^{\prime}\right|\right)$ will belong to $\left[2^{n}, 2^{n+1}\right)$, for some integer $n$, we shall denote

$$
\lambda=\frac{2^{n+1}-|\varepsilon|}{2^{n}} \quad\left(\text { respectively } \lambda^{\prime}=\frac{2^{n+1}-\left|\varepsilon^{\prime}\right|}{2^{n}}\right) .
$$

We can suppose that $\varepsilon$ is smaller than $\varepsilon^{\prime}$ in the lexicographic order. Denote $\delta$ the greatest common ancestor of $\varepsilon$ and $\varepsilon^{\prime}$. And let $d=|\varepsilon|-|\delta|$ (respectively $\left.d^{\prime}=\left|\varepsilon^{\prime}\right|-|\delta|\right)$. 
1) if $2^{n} \leq|\varepsilon|,\left|\varepsilon^{\prime}\right| \leq 2^{n+1}$.

We have,

$$
\begin{gathered}
x_{n, \Psi_{n}(\delta)}^{*} \Pi_{n}\left(f(\varepsilon)-f\left(\varepsilon^{\prime}\right)\right)=\theta\left(\lambda d-\lambda^{\prime} d^{\prime}\right) \\
x_{n+1, \Psi_{n+1}(\delta)}^{*} \Pi_{n+1}\left(f(\varepsilon)-f\left(\varepsilon^{\prime}\right)\right)=\theta\left((1-\lambda) d-\left(1-\lambda^{\prime}\right) d^{\prime}\right) .
\end{gathered}
$$

Hence,

And,

$$
\left\|f(\varepsilon)-f\left(\varepsilon^{\prime}\right)\right\| \geq \frac{\theta\left(d-d^{\prime}\right)}{8} .
$$

$$
\begin{aligned}
-x_{n, \Psi_{n}(\varepsilon)}^{*} \Pi_{n}\left(f(\varepsilon)-f\left(\varepsilon^{\prime}\right)\right) & =\theta \lambda^{\prime} d^{\prime} \\
-x_{n+1, \Psi_{n+1}(\varepsilon)}^{*} \Pi_{n+1}\left(f(\varepsilon)-f\left(\varepsilon^{\prime}\right)\right) & =\theta\left(1-\lambda^{\prime}\right) d^{\prime} .
\end{aligned}
$$

So,

$$
\left\|f(\varepsilon)-f\left(\varepsilon^{\prime}\right)\right\| \geq \frac{\theta d^{\prime}}{8} .
$$

Finally if we distinguish the cases $\frac{d}{2} \leq d^{\prime}$, and $d^{\prime}<\frac{d}{2}$ we obtain:

$$
\left\|f(\varepsilon)-f\left(\varepsilon^{\prime}\right)\right\| \geq \frac{\theta\left(d+d^{\prime}\right)}{24}=\frac{\theta}{24} \rho\left(\varepsilon, \varepsilon^{\prime}\right) .
$$

2) if $2^{n} \leq|\varepsilon| \leq 2^{n+1} \leq 2^{q+1} \leq\left|\varepsilon^{\prime}\right| \leq 2^{q+2}$, or $2^{n} \leq\left|\varepsilon^{\prime}\right| \leq 2^{n+1} \leq 2^{q+1} \leq|\varepsilon| \leq 2^{q+2}$.

If $n<q$,

$$
\left|x_{q+1, \Psi_{q+1}(\delta)}^{*} \Pi_{q+1}\left(f(\varepsilon)-f\left(\varepsilon^{\prime}\right)\right)+x_{q+2, \Psi_{q+2}(\delta)}^{*} \Pi_{q+2}\left(f(\varepsilon)-f\left(\varepsilon^{\prime}\right)\right)\right|=\theta \operatorname{Max}\left(d, d^{\prime}\right)
$$

Hence,

$$
\left\|f(\varepsilon)-f\left(\varepsilon^{\prime}\right)\right\| \geq \frac{\theta}{16} \rho\left(\varepsilon, \varepsilon^{\prime}\right)
$$

If $n=q$ and $|\varepsilon| \leq\left|\varepsilon^{\prime}\right|$,

$$
\left|x_{n+1, \Psi_{n+1}(\varepsilon)}^{*} \Pi_{n+1}\left(f(\varepsilon)-f\left(\varepsilon^{\prime}\right)\right)+x_{n+2, \Psi_{n+2}(\delta)}^{*} \Pi_{n+2}\left(f(\varepsilon)-f\left(\varepsilon^{\prime}\right)\right)\right| \geq \theta d^{\prime} .
$$

So,

$$
\text { If } n=q \text { and }\left|\varepsilon^{\prime}\right|<|\varepsilon| \text {, }
$$

$$
\left\|f(\varepsilon)-f\left(\varepsilon^{\prime}\right)\right\| \geq \frac{\theta}{16} \rho\left(\varepsilon, \varepsilon^{\prime}\right)
$$

$$
\begin{aligned}
& x_{n+1, \Psi_{n+1}(\delta)}^{*} \Pi_{n+1}\left(f(\varepsilon)-f\left(\varepsilon^{\prime}\right)\right)-x_{n+1, \Psi_{n+1}(\varepsilon)}^{*} \Pi_{n+1}\left(f(\varepsilon)-f\left(\varepsilon^{\prime}\right)\right) \\
& \quad+x_{n+2, \Psi_{n+2}(\delta)}^{*} \Pi_{n+2}\left(f(\varepsilon)-f\left(\varepsilon^{\prime}\right)\right)=\theta d .
\end{aligned}
$$

Hence,

$$
\left\|f(\varepsilon)-f\left(\varepsilon^{\prime}\right)\right\| \geq \frac{\theta}{24} \rho\left(\varepsilon, \varepsilon^{\prime}\right)
$$

Finally $T \stackrel{\frac{216}{\theta}}{\hookrightarrow} X$.

Corollary 2.2. $X$ is non super-reflexive if and only if $(T, \rho)$ embeds into $X$. 
Proof. It follows clearly from Bourgain's result [2] and Theorem 1.1.

Remark 2.3. We deduce from the last corollary that the free group with two elements $\mathbb{F}_{2}$ viewed as a metric space through its Cayley graph equipped with the word metric embeds into any non super-reflexive space.

3. Metric characterization of the linear type. First we identify canonicaly $\{-1,1\}^{n}$ with $K_{n}=\{-1,1\}^{n} \times \prod_{k>n}\{0\}$.

Let $p \in[1, \infty)$.

Then we define an other metric on $T=\bigcup K_{n}$ as follows :

$\forall \varepsilon, \varepsilon^{\prime} \in T$,

$$
d_{p}\left(\varepsilon, \varepsilon^{\prime}\right)=\left(\sum_{i=0}^{\infty}\left|\varepsilon_{i}-\varepsilon_{i}^{\prime}\right|^{p}\right)^{\frac{1}{p}} .
$$

The length of $\varepsilon \in T$ can be viewed as $|\varepsilon|=\left(d_{p}(\varepsilon, 0)\right)^{p}$.

The norm $\|\cdot\|_{p}$ on $\ell_{p}$ coincides with $d_{p}$ for the elements of $T$.

We recall now two classical definitions:

Let $X$ and $Y$ be two Banach spaces. If $X$ and $Y$ are linearly isomorphic, the Banach-Mazur distance between $X$ and $Y$, denoted by $d_{B M}(X, Y)$, is the infimum of $\|T\|\left\|T^{-1}\right\|$, over all linear isomorphisms $T$ from $X$ onto $Y$.

For $p \in[1, \infty]$, we say that a Banach space $X$ uniformly contains the $\ell_{p}^{n}$ 's if there is a constant $C \geq 1$ such that for every integer $n, X$ admits an $n$-dimensional subspace $Y$ so that $d_{B M}\left(\ell_{p}^{n}, Y\right) \leq C$.

We state and prove now the following result.

Theorem 3.1. Let $p \in[1, \infty)$.

If $X$ uniformly contains the $\ell_{p}^{n}$ 's then $\left(T, d_{p}\right)$ embeds into $X$.

Proof. We first recall a fundamental result due to Krivine (for $1<p<\infty$ in [8]) and James (for $p=1$ and $\infty$ in [7]).

Theorem 3.2 (James-Krivine). Let $p \in[1, \infty]$ and $X$ be a Banach space uniformly containing the $\ell_{p}^{n}$ 's. Then, for any finite codimensional subspace $Y$ of $X$, any $\epsilon>0$ and any $n \in \mathbb{N}$, there exists a subspace $F$ of $Y$ such that $d_{B M}\left(\ell_{p}^{n}, F\right)<1+\epsilon$.

Using Theorem 3.2 together with the fact that each $\ell_{p}^{n}$ is finite dimensional, we can build inductively finite dimensional subspaces $\left(F_{n}\right)_{n=0}^{\infty}$ of $X$ and $\left(R_{n}\right)_{n=0}^{\infty}$ so that for every $n \geq 0, R_{n}$ is a linear isomorphism from $\ell_{p}^{n}$ onto $F_{n}$ satisfying

$$
\forall u \in \ell_{p}^{n} \quad \frac{1}{2}\|u\| \leq\left\|R_{n} u\right\| \leq\|u\|
$$

and also such that $\left(F_{n}\right)_{n=0}^{\infty}$ is a Schauder finite dimensional decomposition of its closed linear span $Z$. More precisely, if $P_{n}$ is the projection from $Z$ onto $F_{0} \oplus \ldots \oplus F_{n}$ 
with kernel $\overline{\operatorname{Span}}\left(\bigcup_{i=n+1}^{\infty} F_{i}\right)$, we will assume as we may, that $\left\|P_{n}\right\| \leq 2$. We denote now $\Pi_{0}=P_{0}$ and $\Pi_{n}=P_{n}-P_{n-1}$ for $n \geq 1$. We have that $\left\|\Pi_{n}\right\| \leq 4$.

We now consider $\varphi_{n}: T_{n} \rightarrow \ell_{p}^{n}$ defined by

$$
\forall \varepsilon \in T_{n}, \quad \varphi_{n}(\varepsilon)=\sum_{i=1}^{|\varepsilon|} \varepsilon_{i} e_{i},
$$

where $\left(e_{i}\right)$ is the canonical basis of $\ell_{p}^{n}$. The map $\varphi_{n}$ is clearly an isometric embedding of $T_{n}$ into $\ell_{p}^{n}$.

Then we set :

$$
\forall \varepsilon \in T_{n}, \quad f_{n}(\varepsilon)=R_{n}\left(\varphi_{n}(\varepsilon)\right) \in F_{n} .
$$

Finally we construct a map $f: T \rightarrow X$ as follows:

$$
\begin{aligned}
f: T & \rightarrow X \\
\varepsilon & \mapsto \lambda f_{m}(\varepsilon)+(1-\lambda) f_{m+1}(\varepsilon), \text { if } 2^{m} \leq|\varepsilon|<2^{m+1},
\end{aligned}
$$

where,

$$
\lambda=\frac{2^{m+1}-|\varepsilon|}{2^{m}} .
$$

Remark 3.3. We have $\frac{1}{16}|\varepsilon|^{\frac{1}{p}} \leq\|f(\varepsilon)\| \leq|\varepsilon|^{\frac{1}{p}}$.

Like in the proof of Theorem 1.1, we prove that $f$ is 9-Lipschitz using exactly the same computations.

We shall now prove that $f^{-1}$ is Lipschitz. We consider $\varepsilon, \varepsilon^{\prime} \in T$ and assume again that $0<|\varepsilon| \leq\left|\varepsilon^{\prime}\right|$. We need to study two different cases. Again, whenever $|\varepsilon|$ (respectively $\left|\varepsilon^{\prime}\right|$ ) will belong to $\left[2^{m}, 2^{m+1}\right.$ ), for some integer $m$, we shall denote

$$
\left.\lambda=\frac{2^{m+1}-|\varepsilon|}{2^{m}} \text { (respectively } \lambda^{\prime}=\frac{2^{m+1}-\left|\varepsilon^{\prime}\right|}{2^{m}}\right) .
$$

1) if $2^{m} \leq|\varepsilon|,\left|\varepsilon^{\prime}\right|<2^{m+1}$.

$$
\begin{aligned}
d_{p}\left(\varepsilon, \varepsilon^{\prime}\right) \leq & \left\|\lambda \sum_{i=1}^{|\varepsilon|} \varepsilon_{i} e_{i}-\lambda^{\prime} \sum_{i=1}^{\left|\varepsilon^{\prime}\right|} \varepsilon_{i}^{\prime} e_{i}\right\|_{p}+\|(1-\lambda) \sum_{i=1}^{|\varepsilon|} \varepsilon_{i} e_{i} \\
& -\left(1-\lambda^{\prime}\right) \sum_{i=1}^{\left|\varepsilon^{\prime}\right|} \varepsilon_{i}^{\prime} e_{i} \|_{p} \\
\leq & 2\left\|\Pi_{m}\left(f(\varepsilon)-f\left(\varepsilon^{\prime}\right)\right)\right\|+2\left\|\Pi_{m+1}\left(f(\varepsilon)-f\left(\varepsilon^{\prime}\right)\right)\right\| \\
\leq & 16\left\|f(\varepsilon)-f\left(\varepsilon^{\prime}\right)\right\| .
\end{aligned}
$$


2) if $2^{m} \leq|\varepsilon| \leq 2^{m+1} \leq 2^{q+1} \leq\left|\varepsilon^{\prime}\right|<2^{q+2}$.

if $m<q$,

$d_{p}\left(\varepsilon, \varepsilon^{\prime}\right) \leq 2 d_{p}\left(\varepsilon^{\prime}, 0\right)$

$\leq 2\left(\left(1-\lambda^{\prime}\right) d_{p}\left(\varepsilon^{\prime}, 0\right)+\lambda^{\prime} d_{p}\left(\varepsilon^{\prime}, 0\right)\right)$

$\leq 2\left(2\left\|\Pi_{q+2}\left(f(\varepsilon)-f\left(\varepsilon^{\prime}\right)\right)\right\|+2\left\|\Pi_{m+1}\left(f(\varepsilon)-f\left(\varepsilon^{\prime}\right)\right)\right\|\right)$

$\leq 32\left\|f(\varepsilon)-f\left(\varepsilon^{\prime}\right)\right\|$.

if $m=q$,

$$
\begin{aligned}
d_{p}\left(\varepsilon, \varepsilon^{\prime}\right) \leq & \lambda d_{p}(\varepsilon, 0)+\left\|(1-\lambda) \sum_{i=1}^{|\varepsilon|} \varepsilon_{i} e_{i}-\lambda^{\prime} \sum_{i=1}^{\left|\varepsilon^{\prime}\right|} \varepsilon_{i}^{\prime} e_{i}\right\|_{p}+\left(1-\lambda^{\prime}\right) d_{p}\left(\varepsilon^{\prime}, 0\right) \\
\leq & 2\left\|\Pi_{m}\left(f(\varepsilon)-f\left(\varepsilon^{\prime}\right)\right)\right\|+2\left\|\Pi_{m+1}\left(f(\varepsilon)-f\left(\varepsilon^{\prime}\right)\right)\right\| \\
& +2\left\|\Pi_{m+2}\left(f(\varepsilon)-f\left(\varepsilon^{\prime}\right)\right)\right\| \\
\leq & 24\left\|f(\varepsilon)-f\left(\varepsilon^{\prime}\right)\right\| .
\end{aligned}
$$

Finally we obtain that $f^{-1}$ is 32 -Lipschitz, and $T \stackrel{288}{\hookrightarrow} X$.

In the sequel a Banach space $X$ is said to have type $p>0$ if there exists a constant $T<\infty$ such that for every $n$ and every $x_{1}, \ldots, x_{n} \in X$,

$$
\mathbb{E}_{\varepsilon}\left\|\sum_{j=1}^{n} \varepsilon_{j} x_{j}\right\|_{X}^{p} \leq T^{p} \sum_{j=1}^{n}\left\|x_{j}\right\|_{X}^{p},
$$

where the expectation $\mathbb{E}_{\varepsilon}$ is with respect to a uniform choice of signs $\varepsilon_{1}, \ldots, \varepsilon_{n} \in$ $\{-1,1\}^{n}$.

The set of $p$ 's for which $X$ contains $\ell_{p}^{n}$ 's uniformly is closely related to the type of $X$ according to the following result due to Maurey, Pisier [10] and Krivine [8], which clarifies the meaning of these notions.

Theorem 3.4 (Maurey-Pisier-Krivine). Let $X$ be an infinite-dimensional Banach space. Let

$$
p_{X}=\sup \{p ; \mathrm{X} \text { is of type } \mathrm{p}\},
$$

Then $X$ contains $\ell_{p}^{n}$ 's uniformly for $p=p_{X}$.

Equivalently, we have

$$
p_{X}=\inf \left\{p ; \mathrm{X} \text { contains } \ell_{\mathrm{p}}^{\mathrm{n}}, s \text { uniformly }\right\} .
$$

We deduce from Theorem 3.1 two corollaries.

Corollary 3.5. Let $X$ a Banach space and $1 \leq p<2$.

The following assertions are equivalent:

i) $p_{X} \leq p$ 
ii) $X$ uniformly contains the $\ell_{p}^{n}$ 's.

iii) the $\left(T_{n}, d_{p}\right)$ 's uniformly embed into $X$.

iv) $\left(T, d_{p}\right)$ embeds into $X$.

Proof. ii) implies $i$ ) is obvious.

i) implies ii) is due to Theorem 3.2 and the work of Bretagnolle, DacunhaCastelle and Krivine [4].

For the equivalence between $i$ ) and $i$ ii) see the work of Bourgain, Milman and Wolfson [3] and Krivine [8].

$i v$ ) implies iii) is obvious.

And $i i$ ) implies $i v$ ) is Theorem 3.1.

Corollary 3.6. Let $X$ be an infinite dimensional Banach space, then $\left(T, d_{2}\right)$ embeds into $X$.

Proof. This corollary is a consequence of the Dvoretsky's Theorem [6] and Theorem 3.1.

\section{References}

[1] I. Aharoni, Every separable metric space is Lipschitz equivalent to a subset of $c_{0}^{+}$. Israel J. Math. 19, 284-291 (1974).

[2] J. Bourgain, The metrical interpretation of super-reflexivity in Banach spaces. Israel J. Math. 56, 221-230 (1986).

[3] J. Bourgain, V. Milman, and H. Wolfson, On type of metric spaces. Trans. Amer. Math. Soc. 294(1), 295-317 (March 1986).

[4] J. Bretagnolle, D. Dacunha-Castelle, and J. L. Krivine, Lois stables et espaces $L^{p}$. Ann. Instit. H. Poincaré 2, 231-259 (1966).

[5] J. Diestel, Sequences and Series in Banach Spaces, Springer-Verlag, 1984.

[6] A. Dvoretzky, Some results on convex bodies and Banach spaces. Proc. Internat. Sympos. Linear Spaces (Jerusalem, 1960) 123-160.

[7] R. C. James, Super-reflexive spaces with bases. Pacific J. Math. 41, 409-419 (1972).

[8] J. L. KRivine, Sous-espaces de dimension finie des espaces de Banach réticulés. Ann. of Math. 104(2), 1-29 (1976).

[9] J. Lindenstrauss and L. Tzafriri, Classical Banach Spaces I, Springer-Verlag, Berlin, 1977.

[10] B. Maurey And G. Pisier, Séries de variables aléatoires vectorielles indépendantes et propriétés géométriques des espaces de Banach. Studia Math. 58, 45-90 (1976).

[11] M. Mendel and A. NAOR, Metric cotype, arXiv:math.FA/0506201 v3 29 Apr 2006. 
[12] G. Pisien, Factorization of Linear Operators and Geometry of Banach Spaces. CBMS Regional Conference Series in Mathematics 60.

[13] M. RiBE, Existence of separable uniformly homeomorphic non isomorphic Banach spaces. Israel J. Math. 48, 139-147 (1984).

Florent Baudier, Laboratoire de Mathématiques, UMR 6623, Université de FrancheComté, 25030 Besançon, cedex, France

e-mail: florent.baudier@univ-fcomte.fr

Received: 2 August 2006

Revised: 10 April 2007 